\title{
Single-Stage Treatment of Extensive Aortic Pathology Using the Frozen Elephant Trunk Procedure
}

\author{
Petar Risteski, ${ }^{1}$ Vasil Papestiev, ${ }^{2}$ Sasko Jovev, ${ }^{2}$ Stefanija Hadzievska, ${ }^{2}$ Nadica Mehmedovic, ${ }^{2}$ Nikola Lazovski, ${ }^{2}$ \\ Zan Zimbakov, ${ }^{3}$ Venko Filipce, ${ }^{4}$ Marjan Shokarovski ${ }^{2}$
}

\begin{abstract}
${ }^{1}$ Department of Thoracic and Cardiovascular Surgery, University Hospital Frankfurt, Goethe University, Frankfurt, Germany; ${ }^{2}$ University Clinic for Cardiac Surgery, Skopje, North Macedonia; ${ }^{3}$ University Clinic for Cardiology, Skopje, North Macedonia; ${ }^{4}$ University Clinic for Neurosurgery, Skopje, North Macedonia
\end{abstract}

\section{ABSTRACT}

Background: Extensive pathology involving the aortic arch and descending aorta traditionally has been treated with two open procedures. We report our institutional experience with a single stage frozen elephant trunk procedure for treatment of extensive aortic pathology.

Methods: Between June 2018 and October 2019, nine patients (eight males, $89 \%$, mean age $61 \pm 6$ years) with extensive aortic pathology were operated using the frozen elephant trunk procedure. Five (56\%) patients underwent primary operation for chronic arch and proximal descending aneurysm in two (22\%) patients, chronic type B aortic dissection in two (22\%) patients and penetrating aortic ulcer in one (11\%) patient. The other four $(44 \%)$ patients received reoperative surgery for chronic post-dissection aneurysms. For organ protection during the aortic arch procedure, we used selective antegrade cerebral perfusion and mild systemic hypothermia at $28^{\circ} \mathrm{C}$.

Results: Early mortality was not observed. A single (11\%) patient developed focal stroke. Unilateral vocal cord palsy was present in two (22\%) patients. Spinal cord injury was not observed. Reexploration for bleeding was required in two (22\%) patients. Prolonged ventilation, liver and kidney failure as well as cardiac morbidity were not observed. Two patients (22\%) with anticipated Endoleak type $\mathrm{Ib}$ received TEVAR extension at follow up. Mid-term mortality was observed in two (22\%) patients, due to pneumonia.

Conclusion: The frozen elephant trunk procedure can be used for a single-stage treatment of patients with extensive aortic pathology, due to chronic degenerative aneurysms or post-dissection aneurysms involving the aortic arch and the descending aorta, with acceptable mortality and morbidity.

\section{INTRODUCTION}

The treatment of aortic aneurysms traditionally has involved resection of the diseased aortic segment and

Received April 15, 2020; received in revised form fune 27, 2020; accepted fune 29, 2020.

Correspondence: Prof. Dr. Sasko Fovev, University Clinic for Cardiac Surgery, Majka Tereza 17/8, 1000 Skopje, North Macedonia; +389-2-3147-939; fax +389-2-3211-218 (e-mail: jovev99@yahoo.com). replacement with a vascular prosthesis [Erbel2014; Luehr2018]. Pathology involving two or more anatomic segments of the thoracic aorta, like the aortic arch and proximal descending aorta, traditionally has been treated with two open procedures or stages, initially through a median sternotomy followed by lateral thoracotomy later on, which altogether pose a tremendous burden of invasiveness for the patients [Estrera 2014].

The frozen elephant trunk (FET) procedure, combining conventional open aortic repair and endovascular stenting, has been introduced, promptly popularised and recently recommended as a single-stage treatment of choice for patients with extensive aortic disease [Risteski 2017; Grabenwoger 2012; Zierer 2017].

In this paper, we report our institutional experience with the frozen elephant trunk procedure for treatment of extensive aortic pathology.

\section{MATERIALS AND METHODS}

From June 2018 to October 2019, nine patients (eight males, $89 \%$, mean age $61 \pm 6$ years) with extensive aortic pathology were operated using the FET procedure. The indication for primary surgery in five $(56 \%)$ patients included chronic distal arch and proximal descending aneurysm in two (22\%) patients, chronic type B aortic dissection not amendable to total endovascular repair (TEVAR) in two (22\%) patients and penetrating aortic ulcer in one $(11 \%)$ patient. The other four (44\%) patients received reoperative surgery for chronic post-dissection aneurysms involving the aortic arch and the descending aorta (Figure 1).

Table 1 outlines the preoperative patients demographics and comorbidities (Table 1). The most common preoperative comorbidity was systemic hypertension present in eight (89\%) patients. Previous stroke was present in three $(33 \%)$ patients. Four patients had undergone previous surgery for acute type A aortic dissection. Two of them were reoperated on the aortic root during a planned reoperative procedure elsewhere, years prior to the FET procedure in our unit. Thus, the FET procedure was the second reoperative surgery in these two patients.

Preoperative diagnostic workup included computed tomography (CT) scan of the aorta in every patient to confirm the indication and to plan the operative treatment, with 


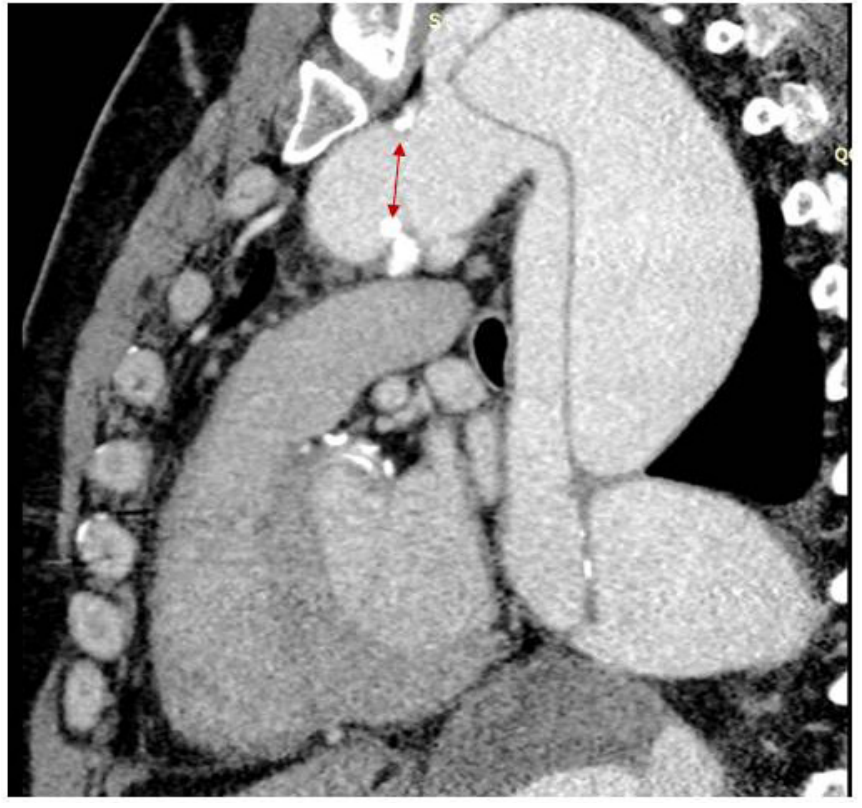

Figure 1. Chronic post-dissection aneurysm of the distal aortic arch and the proximal descending aorta in a patient who previously underwent replacement of the ascending aorta and the proximal hemiarch (red double arrow indicate the Teflon felt strip reinforcement of the distal anastomosis) initially, followed by aortic root replacement several years later.

emphasis on the extent of resection of the aortic pathology and the distal extent of the exclusion of the aneurysm. The distal landing zone for the stent portion of the hybrid prosthesis was identified in the descending aorta and sized at the level of the upper pulmonary veins. As a rule of a thumb, $10-15 \%$ oversizing was used in patients with aneurysms to determine the diameter of the stent portion of the hybrid prosthesis in order to provide adequate sealing and avoid postoperative type Ib Endoleak. In patients with chronic dissection, where the perimeter of the true lumen was used to provide the area and then the diameter of the true lumen, $10-20 \%$ oversizing was employed. Preoperative diagnostic workup was completed with coronary angiography, transthoracic and transesophageal echocardiography (TEE), lung function assessment and carotid doppler exam.

The local ethics committee approved the study, and every patient signed informed consent. The clinical data management was in accordance with the declaration of Helsinki.

Operative technique: All patients received through an arterial sheath (TFA Glidesheath 5 Fr., Terumo Deutschland GmbH, Eschborn, Germany) a single stiff guide wire (Amplatz super stiff $0.035 \mathrm{GW}, 145 \mathrm{~cm}$ in length, Boston Scientific, Marlborough, Massachusetts, USA) placed through a puncture of the femoral artery, either in the operating room in case of chronic degenerative aneurysm or in the catheterisation lab in case of chronic post-dissection aneurysm. The wire was protected with a pigtail catheter (Radifocus Optitorque PIG 5 Fr., $110 \mathrm{~cm}$ in length, Terumo Deutschland GmbH, Eschborn, Germany) to prevent iatrogenic aortic injury,
Table 1. Preoperative patients demographics and comorbidities

\begin{tabular}{ll}
\hline Systemic hypertension (n, \%) & $8(89)$ \\
Diabetes mellitus (n, \%) & $1(11)$ \\
Coronary artery disease (n, \%) & $4(44)$ \\
Chronic obstructive pulmonary disease (n, \%) & $4(44)$ \\
Chronic renal failure (n, \%) & $3(33)$ \\
Peripheral vascular disease (n, \%) & $3(33)$ \\
Cerebrovascular disease (n, \%) & $2(22)$ \\
Tobacco abuse (n, \%) & $3(33)$ \\
Previous coronary artery bypass grafting (n, \%) & $2(22)$ \\
Previous aortic valve replacement (n, \%) & $4(44)$
\end{tabular}

and the placement in the distal aortic arch was confirmed with either TEE or fluoroscopy.

After a median sternotomy and administration of heparin (400 units/kg), the right axillary artery was directly cannulated in eight patients in the deltopectoral groove with an 18-20 Fr. flexible arterial cannula (EOPA, Medtronic, Dublin, Ireland). In a single patient, alternatively, the right common carotid artery was cannulated through an $8 \mathrm{~mm}$ side arm polyester graft. For venous drainage, following pericardiotomy, the right atrium was cannulated with a two-stage cannula (MC2 Cannula, Medtronic, Dublin, Ireland). The brachiocephalic, left common carotid and left subclavian artery were dissected free and encircled with elastomer bands. The aortic arch was circumferentially dissected free up to zone 2. Further dissection of the aortic arch was not performed in order to preserve the left recurrent laryngeal nerve. For the same reason, the distal anastomosis with the hybrid prosthesis was planned in zone 2. Debranching of the left subclavian artery was performed with an $8 \mathrm{~mm}$ polyester graft.

This $8 \mathrm{~mm}$ graft was then cannulated with an $18 \mathrm{~F} \mathrm{flex-}$ ible cannula, deaired and connected to the arterial return line of the cardiopulmonary bypass, during cooling down of the patient to the target temperature of $28^{\circ} \mathrm{C}$. Afterward, an antegrade uninterrupted flow through the left subclavian artery was established to have good perfusion of the spinal cord, during the period of moderate hypothermic lower body circulatory arrest.

After cardioplegic arrest, the ascending aorta was resected, if required, and once the target temperature was reached, both elastomer bands used to temporarily occlude the ostia of the brachiocephalic trunk and the left common carotid artery were tightened. The flow on the pump was reduced, and the aortic clamp was released. A sucker was placed in the descending aorta and once bloodless field was secured, the aortic arch was resected up to zone 2 (Figure 2).

Next, the elastomer loop snared around the left common carotid artery was temporarily loosened and a flexible balloontipped arterial cannula was placed inside the vessel for additional perfusion of the left cerebral hemisphere. This arterial line was simply connected as a side branch of the arterial circuit. Selective antegrade cerebral perfusion was conducted with a perfusate temperature of $28^{\circ} \mathrm{C}$ in a pressure-controlled 


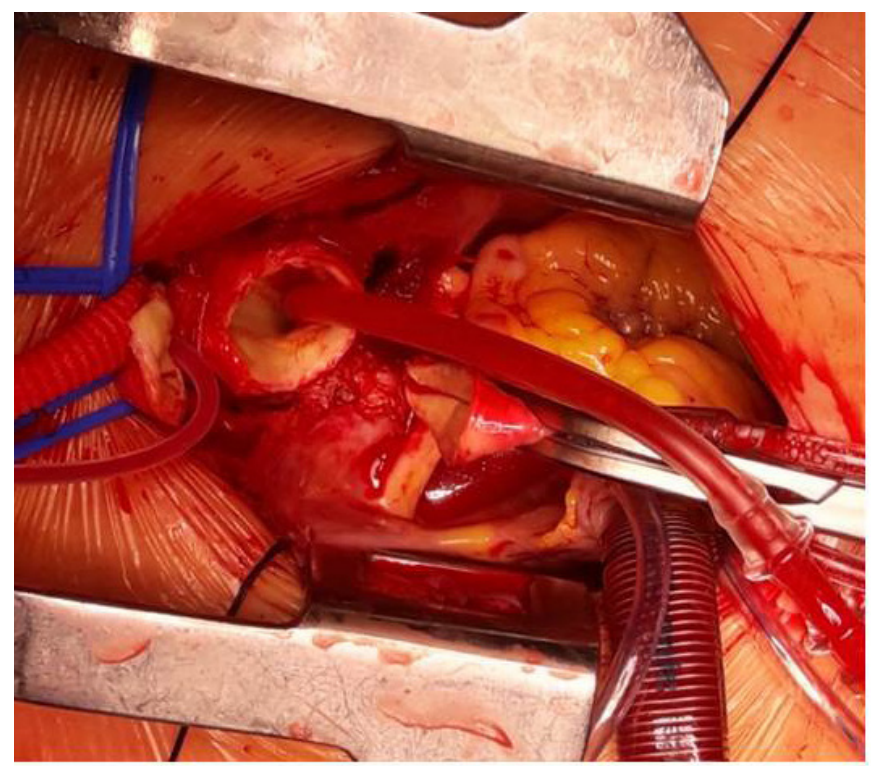

Figure 2. Intraoperative photo of the operative situs as seen through a partial upper ministernotomy. After resection of the aortic arch, the proximal descending aorta was exposed. A sucker was placed in the descending aorta to provide for a bloodless field. Note the yellow arrow pointing on the $8 \mathrm{~mm}$ polyester graft used to debranch the left subclavian artery.

manner. The perfusion pressure was controlled at the pump unit and kept at 80 to $90 \mathrm{mmHg}$ as measured on the main pump and corresponded with a $60 \mathrm{mmHg}$ pressure as measured with the left radial artery catheter. Neurovascular monitoring consisted of cerebral saturation assessment using nearinfrared spectroscopy over both frontal cerebral cortexes. We used the INVOS (Somanetics Corporation, MI, USA), which measures the regional oxygen saturation $(\mathrm{rSaO} 2)$ in percent, representing a valuable neurovascular monitoring tool.

Using the guide wire, the hybrid prosthesis (Evita open plus, CryoLife JOTEC GmbH, Hechingen, Germany) was advanced through the opened distal aortic arch and further deployed under direct vision. The collar of the hybrid prosthesis was sutured in zone 2 using a $120 \mathrm{~cm}$ long 3/0 polypropylene mounted on a $\mathrm{SH}$ needle in continuous suture technique. Afterward, the invaginated polyester part of the hybrid prosthesis was retracted from the stent and used to replace the aortic arch. The brachiocephalic and left common carotid artery were re-implanted using the Carrel island technique with a continuous polypropylene $4 / 0$ suture. Following deairing of the prosthesis, the graft was clamped, the perfusion of the whole body was resumed via the brachiocephalic trunk, rewarming was initiated, and the proximal anastomosis of the hybrid prosthesis with the ascending aorta was performed in a usual fashion. The procedure was completed with the proximal anastomosis of the debranched left subclavian artery graft on the ascending aorta (Figure 3).

Definitions of outcomes, data analysis and follow up: A transient neurologic dysfunction (TND) was defined as the presence of a reversible postoperative motor deficit, confusion, agitation, or transient delirium. The computed
Table 2. Intraoperative and early postoperative results

\begin{tabular}{lc}
\hline Cardiopulmonary bypass time (min.) & $172 \pm 35$ \\
Aortic cross-clamp time (min.) & $62 \pm 29$ \\
Antegrade cerebral perfusion time (min.) & $32 \pm 14$ \\
Concomitant coronary artery bypass grafting (n, \%) & $2(22)$ \\
Ventilation time (hours) & $15 \pm 2$ \\
Intensive care unit stay (days) & $4 \pm 1$ \\
Hospital stay (days) & $21 \pm 11$
\end{tabular}

tomography findings were required to be normal, with resolution of all symptoms before discharge. A permanent neurologic deficit (PND) was defined as the presence of either new focal (stroke) or global (coma) permanent neurologic dysfunction. Early mortality was defined as death during the initial hospitalization or within 30 days postoperatively for discharged patients. Respiratory insufficiency was defined in patients requiring a repeat intubation and ventilation. Prolonged ventilation was defined as that lasting more than seven days. Reexploration for bleeding was defined as significant bleeding requiring explorative rethoracotomy for hemostasis. Acute renal failure was defined when the glomerular filtration rate was less than $60 \mathrm{ml} / \mathrm{min} / 1.73 \mathrm{~m}^{2}$. Positive aortic remodeling was assessed at the level of the descending aorta and defined as complete thrombosis of the excluded aortic aneurysm with radiologic evidence of regression of the aneurysm, or in patients with dissection - as thrombosis and regression of the false lumen.

All preoperative, perioperative, and postoperative data were collected in a central database for purposes of this retrospective single-center cohort observation. Categorical parameters were summarized as absolute numbers and percentages. Continuous data are shown as mean $\pm \mathrm{SD}$ or median + interquartile range.

Survivors were followed, during a period of 108 patientmonths, systematically in our out-patient clinic with physical examination and at least an annual CT scan of the aorta. No patient was lost to follow up. Mean follow up amounted $12 \pm$ 3.5 months (range 4-20 months) and was complete.

\section{RESULTS}

Early results: Table 2 summarizes the early results (Table 2). Early mortality was not observed. Six (67\%) patients demonstrated early postoperative delirium, which completely resolved after several days. A single (11\%) patient developed focal PND; global PND was not observed. Unilateral vocal cord palsy was present in two (22\%) patients. Partial or complete spinal cord injury was not observed.

Reexploration was required in two $(22 \%)$ patients, for pericardial effusion in one and persistent bleeding in another. Respiratory insufficiency was present in three $(33 \%)$ patients, who required repeat intubation. Prolonged ventilation, liver and kidney failure as well as cardiac morbidity were not observed. 


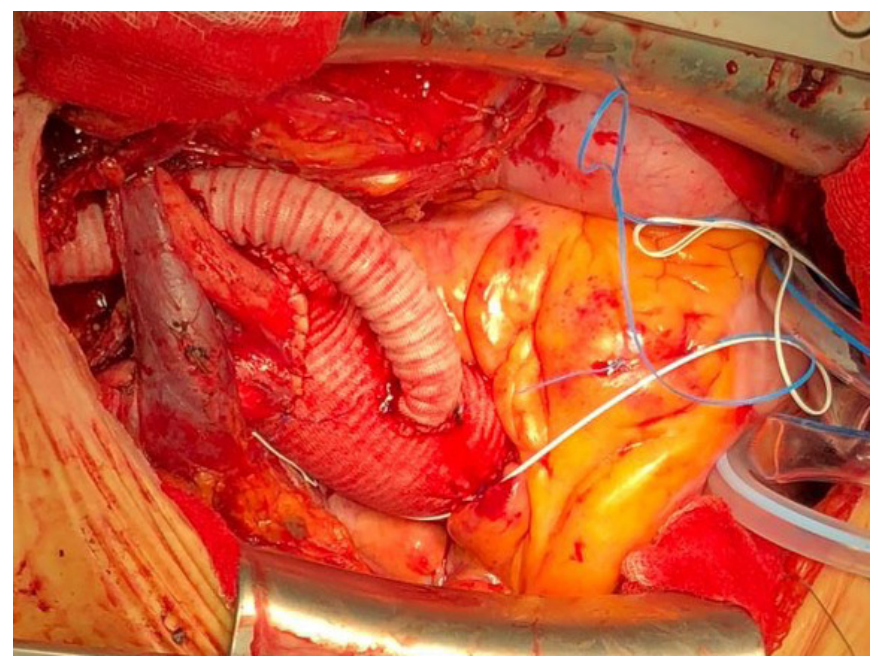

Figure 3. Intraoperative photo of the completed aortic repair and the debranching of the left subclavian artery.

Mid-term outcomes: Two patients died during follow up. The first patient experienced out-of-hospital arrest on postoperative day 60 due to pneumonia-induced respiratory insufficiency. The second patient underwent a peripheral vascular intervention and developed severe respiratory insufficiency as a complication of ventilator-associated pneumonia on postoperative day 122 , and eventually expired due to septic shock. Both patients were morbidly obese with advanced chronic obstructive pulmonary disease.

Radiologic follow up revealed positive aortic remodeling in all patients except two, where an Endoleak type $\mathrm{Ib}$ was present (Figure 4). This Endoleak was anticipated in both patients, as the length of the aneurysm being treated exceeded the maximal stent length available. Both patients received a TEVAR extension to resolve the Endoleak, in both instances an unremarkable postoperative course was observed.

The vocal cord palsy in both patients persisted beyond six months. Apart of hoarseness, it has not limited the lifestyle of the patients.

\section{DISCUSSION}

Aortic aneurysms involving the distal aortic arch and a variable extent of the descending aorta present a significant challenge for the aortic surgeon.

Traditionally, these aneurysms have been treated in two operative procedures or stages. The first stage involved resection of the aortic arch portion of the disease and replacement with a tube graft and usually was performed through a median sternotomy, using a heart-lung machine and deep hypothermic circulatory arrest. Following an interval of several weeks up to months, a second procedure was staged and involved replacement of the descending aorta through a second large incision, a lateral thoracotomy, again using the heart-lung machine to provide for perfusion on the downstream aorta. Technical difficulties frequently were encountered, during

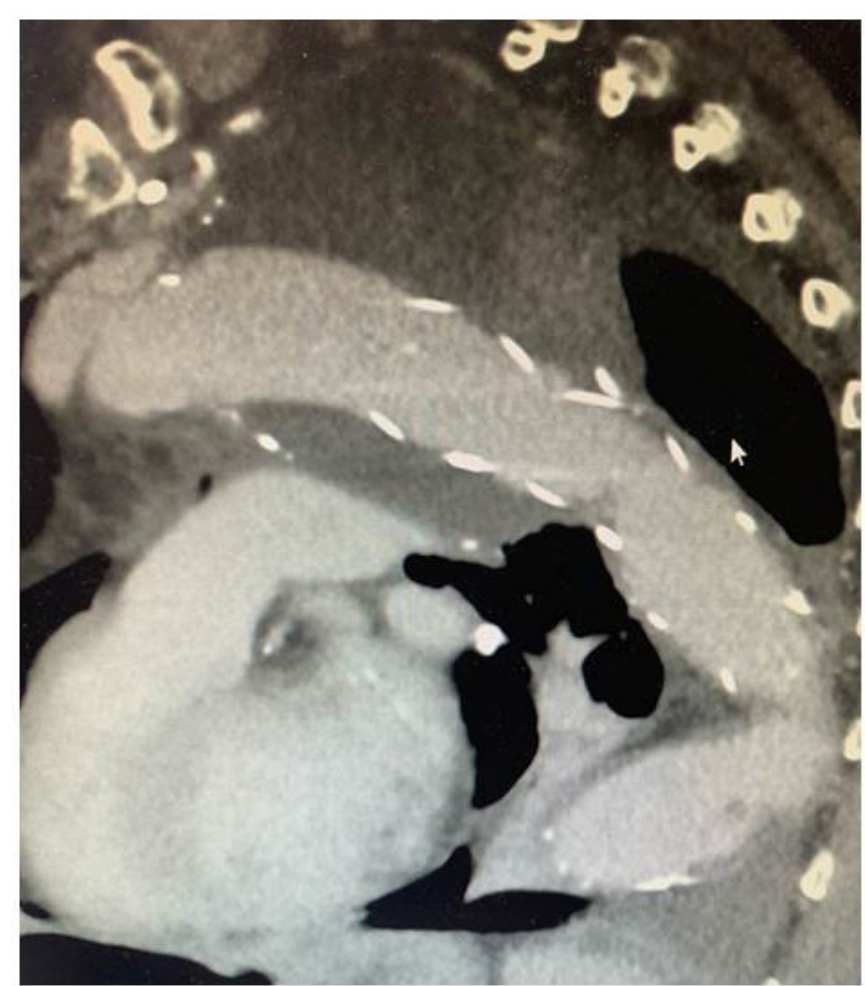

Figure 4. Postoperative CT scan of the aorta, demonstrating complete exclusion of the distal arch/proximal descending aortic aneurysm with thrombosis of the periprosthetic aneurysm sac and sufficient sealing in the distal landing zone.

the second stage, and included troublesome exposure of the distal aortic arch; a hostile region impacted with dense adhesions following the first stage.

In order to improve these outcomes, several advances and modifications have been reported. Hans Borst described in 1983 the conventional elephant trunk technique which included, in addition to a total arch replacement, leaving a tube graft (during the first stage) hanging free in the descending aorta [Borst 1983]. During the second procedure, the graft was clamped and used to anastomose the descending aortic graft without going up to the arch and working in a previously operated region. Even though it made the operation easier for the surgeon, the patient still had to undergo two major procedures, and the outcomes did not considerably improve [Shrestha 2014; Etz 2008]. Early mortality with the first stage was reported in a range from $7-17 \%$ from centers of excellence [Borst 1983; Shrestha 2014]. For those who decided to undergo a second stage operation (reported return rates varied between 44-62\%), early mortality was in the range of 7\% for the complete procedure [Shrestha 2014, Etz 2008]. The invasiveness of both procedures was considerable, and this approach traditionally has been offered only to suitable low-risk younger patients without serious comorbidities. Even then, the cumulative mortality of both procedures and the interval mortality was in the range of over 30\% [Shrestha 2014; Etz 2008]. Other authors reported 
single-stage operative treatment combining both incisions, the sternotomy, and the thoracotomy, but this strategy was not accepted in the surgical community as the invasiveness of both incisions, the extent and rate of the left-sided pulmonary complications and the functional impairment following these procedures were unacceptably high [Doss 2003].

With the advent of the TEVAR procedures, the second stage after conventional elephant trunk could be done endovascularly, avoiding the complications from the open second stage procedure. Lower morbidity and mortality were reported using this approach [Castrovinci 2015].

Another approach is a two-stage procedure of arch debranching first, done with sternotomy, followed by endovascular repair of the diseased arch and descending aorta [De Rango 2014], but long-term results are not available at this time.

The frozen elephant trunk (FET) operation has been developed to overcome all of the obstacles and disadvantages of the previous techniques in the treatment of complex thoracic aortic diseases through the combination of surgical and endovascular techniques. It provides for the treatment to be completed through a single incision, usually median sternotomy, even though a less invasive approach like an upper mini sternotomy also may be used [Risteski 2017]. The hybrid prosthesis includes two components sutured together: a woven vascular graft and a stent. The grafted part is 50-70 $\mathrm{mm}$ long, made of polyester fabric, and offers zero-porosity. The stent part, being 130-160 mm long, treats the surgically inaccessible part of the thoracic aorta through a single incision like median sternotomy. It also provides for an excellent landing zone for future TEVAR procedure, if necessary [Borst 1983; Etz 2008].

As with other complex aortic procedures, organ protection is mandatory for optimal outcome. We used mild systemic hypothermia at $28^{\circ} \mathrm{C}$ and bilateral antegrade cerebral perfusion in a pressure-controlled fashion. Several groups have reported improved patient outcomes using this strategy [Zierer 2017; Preventza 2015]. We also believe that bilateral antegrade cerebral perfusion is mandatory because of the low incidence of stroke and the possibility to extend the operative time in complicated cases. It also gives us the opportunity to employ mild systemic hypothermia at $28^{\circ} \mathrm{C}$ with less bleeding complications. Separate cannulation and perfusion of the left subclavian artery provides for improved spinal cord protection, and lower incidence of paraplegia, even with longer times of lower body circulatory arrest. All patients received controlled perfusion of the left subclavian artery, and spinal cord injury was not observed in this cohort.

Clinical outcomes have considerably improved with the use of the frozen elephant trunk technique primarily related to the optimization of the preoperative planning and the improvement of the intraoperative organ protection strategy (Shrestha 2015 is freezing, Shrestha 2015 recommendation). Nowadays, this treatment can be offered to the patient with single digit mortality or neurologic morbidity rate [Zierer 2017].
REFERENCES

Borst HG, Walterbusch G, Shaps D, et al. 1983. Extensive aortic replacement using "elephant trunk" prosthesis. Thorac Cardiovasc Surg 31:37-40.

Castrovinci S, Murana G, de Maat GE, et al. 2015. The classic elephant trunk technique for staged thoracic and thoracoabdominal aortic repair: long-term results. Thorac Cardiovasc Surg 149(2):416-22.

De Rango P, Cao P, Ferrer C, et al. 2014. Aortic arch debranching and thoracic endovascular repair. J Vasc Surg 59(1):107-14.

Doss M, Woehleke T, Wood JP, Martens S, Greinecker GW, Moritz A. 2003. The clamshell approach for the treatment of extensive thoracic aortic disease. J Thorac Cardiovasc Surg 126(3):814-7.

El-Sayed Ahmad A, Risteski P, Ay M, Papadopoulos N, Moritz A, Zierer A. 2019. Moderate hypothermic circulatory arrest $\left(\geq 28^{\circ} \mathrm{C}\right)$ with selective antegrade cerebral perfusion for total arch replacement with frozen elephant trunk technique. Thorac Cardiovasc Surg 67(5):345-350.

Erbel R, Aboyans V, Boileau C, et al. 2014. 2014 ESC Guidelines on the diagnosis and treatment of aortic diseases. European Heart Journal 35: 2873-2926.

Estrera AL, Sandhu HK, Miller CC, et al. 2014. Repair of extensive aortic aneurysms: a single center experience using elephant trunk technique over 20 years. An Surg 260(3):510-6

Etz CD, Plestis KA, Kari FA, et al. 2008 Staged repair of thoracic and thoracoabdominal aortic aneurysms using the elephant trunk technique: a consecutive series of 215 first stage and 120 complete repairs. Eur J Cardiothorac Surg 34:605-615.

Grabenwoger M, Alfonso F, Bachet J, et al. 2012. Thoracic Endovascular Aortic Repair TEVAR for treatment of aortic diseases: a position statement from European Association for Cardio-Thoracic Surgery EACTS and the European Society of Cardiology ESC, in collaboration with European Association of Percutaneous Cardiovascular Interventions EAPCI. EUR J Cardiothorac Surg 42(1);17-24.

Luehr M, Peterss S, Zierer A, Pacini D, et al. 2018. Aortic events and reoperations after elective aortic arch surgery: incidence, surgical strategies and outcomes. Eur j Cardiothorac Surg 1;53(3):519-524.

Preventza O, Price MD, Simpson KH, et al. 2015. Hemiarch and total arch surgery in patients with previous repair of acute type I aortic dissection. Ann Thorac Surg 100:833-9.

Risteski P, El-Sayed Ahmad A, Monsefi N, et al. 2017. Minimally invasive aortic arch surgery: Early and late outcomes Int J Surg 45 113-117.

Shrestha M, Bachet J, Bavaria J, et al. 2015. Current status and recommendations for use of the frozen elephant trunk technique: a position paper by the Vascular Domain of EACTS. Eur J Cardiothorac Surg 47(5):759-69.

Shrestha M, Beckmann E, Krueger H, et al. 2015. The elephant trunk is freezing: The Hannover experience. J Thorac Cardiovasc Surg 149(5):1286-93.

Shrestha M, Martens A, Krüger H, et al. 2014. Total aortic arch replacement with the elephant trunk technique: single-center 30-year results. Eur J Cardiothorac Surg 45:289-96.

Zierer A, El Sayed Ahmad A, Papadopoulos N, et al. 2017. Fifteen years of surgery for acute type A dissection in moderate to mild systemic hypothermia Eur J Cardiothorac Surg 51 (1): 97-103. 